\title{
Isolation and Characterization Compounds From Hexane and Ethyl Acetate Fractions of Peperomia pellucida L.
}

\author{
Sri Hartati ${ }^{1^{*}}$, Marissa Angelina ${ }^{1}$, Indah D. Dewiyanti ${ }^{1}$, Lia Meiliawati ${ }^{1}$
}

${ }^{1}$ Research Centre for Chemistry Indonesia Institute of Sciences, Kawasan Puspiptek Serpong,

Tangerang Selatan, 15314 Indonesia

\begin{abstract}
Peperomia pellucida was used traditionally in Indonesia for health treatment: wounds, boils, pimples, abscesses, abdominal pain, colic, gout, kidney, rheumatic pain, fatigue headache, furuncles, conjunctivitis and anti dermatogenic and also for dengue treatment. The isolation compounds from hexane and ethyl acetate fractions of Peperomia pellucida L. are conducted by maceration of the dry herbs sample with methanol and partition with hexane, ethyl acetate, butanol and water. The hexane and ethyl acetate fractions were fractionated by gravitation column chromatography and eluted successively with hexane, ethyl acetate and methanol by the gradient. The structure was elucidated base on spectroscopy data of NMR proton and carbon for one and two dimension, LCMS and FT-IR. The isolation founded three compounds are stigmasterol, analogue of pheophytin and $\beta$-sitosterol$D$-glucopyranoside.
\end{abstract}

Keywords: analogue of pheophytin, Peperomia pellucida, stigmasterol, and $\beta$-sitosterol-D-glucopyranoside

\section{INTRODUCTION}

Peperomia pellucida (L.) is belonging Piperaceae, in Indonesia (West Java) known as "Katumpangan air". Peperomia pellucida is ones of folk medicine. It is a herbaceous plant with succulent stems, shiny, heartshaped, freshly leaves and tiny. The whole herb is used as an emollient, diuretic and to control cough and cardiac arrhythmia, boils and skin wounds, eyes inflammation [1, 2]. Uses in traditionally medicine for wounds, boils, pimples, abscesses, abdominal pain, colic, gout, kidney, rheumatic pain, fatigue headache, furuncles, conjunctivitis and anti dermatogenic [3, 4]. Pharmacology properties of P. Pellucida: the analgesic properties of the plant seem to be related to its effect on prostaglandin synthesis [5]. The aqueous extract inhibited an anti-inflammatory activity in the carrageenan test [6]. $P$. pellucida finds its use as a potential source of functional foods [7]. P. pellucida leaf extract possessed anticancer activities against human breast adenocarcinoma (MCF-7) [9]. It was also reported about five new compounds peperomins $\mathrm{A}, \mathrm{B}, \mathrm{C}$, and E, 7,8-trans-8,8'-trans-7',8'-cis-7,7'-bis(5-methoxy-

\footnotetext{
${ }^{*}$ Corresponding author:

Sri Hartati

Research Centre for Chemistry Indonesia Institute of Sciences Serpong, Tangerang Selatan, 15314, Indonesia

E-mail: elzariana@yahoo.com
}

3,4-methylenedioxyphenyl)-8-acetoxymethyl-8'-hydroxymethyltetrahdrofuran, 7,8-trans-8,8'-trans-7',8'-cis-7(5-methoxy-3,4-methylenedeoxyphenyl)-7'-(4-hydro-xi3,5-dimethoxyphenyl)-8,8'-diacetoxymethyl tetrahydrofuran, Pellucidin A, have been isolated from the aerial part of $P$. pellucida [10]. The oil of $P$. pellucida contained dillapiole (55.3\%), (E)-caryophyllene (14.3\%) and carotol (8.1\%) [11]. Rojas-Martinez. et al., 2013 [12] reported that dillapiole was identified as the most active compound in the dichloromethane extract and also reported the gastro protector activity of dillapiole. Ethanolic extract of $P$. pellucida accelerates fracture repairment in rats via stimulatory effects on osteoblast differentiation and mineralisation [13]. In this research have been done an isolation of others compounds which furthermore research will testing for the bioactivity.

\section{MATERIALS AND METHODS Plant Materials}

The raw material of Peperomia pellucida L. was collected from around of Puspiptek Serpong. The voucher specimen was identified in a research center for biology Bogor Indonesia Institute of Sciences, and the specimen was deposited in Herbarium Bogorience research center for biology Bogor. 


\section{Chemical Materials}

Technical organic solvents are ethanol, methanol, ethyl acetate, $\mathrm{n}$-hexane and $\mathrm{n}$-butanol are destiled. Silica gel $\mathrm{G}_{60}(0,062-0,2000 \mathrm{~mm}) \mathrm{E}$ Merck 1.07734, Silica gel G60 $(0,2-0,5 \mathrm{~mm})$ E Merck 1. 07733, Silica Gel G60 $\mathrm{F}_{254}$ E Merck 1.07730, TLC silica gel $60 \mathrm{~F}_{254}$, aluminium sheets E. Merck 1.05554.0001. Sephadex LH-20 Amersham.

\section{Extraction and Isolation}

Peperomia pellucida L. were collected around of puspiptek Serpong South Tangerang. Air dried of herbs $P$. pellucida $(0.988 \mathrm{~kg})$ were extracted exhaustively with $95 \%$ aqueous of ethanol $(5 \times 5 \mathrm{~L})$ at room temperature. The ethanol extract was concentrated in vaquo to yield a dark green semi solid (103 g). $90 \mathrm{~g}$ of ethanol extract was suspended in aqueous $(750 \mathrm{ml})$ and partitioned sequentially in three different solvents, $\mathrm{n}$ hexane $(4 \times 750 \mathrm{ml})$, ethyl acetate $(4 \times 750 \mathrm{ml})$ and $\mathrm{n}$ butanol $(4 \times 750 \mathrm{ml})$, to fractionate polar and nonpolar compounds.

The organic phases were concentrated to yield residues with $28 \mathrm{~g} n$-hexane extract, $3 \mathrm{~g}$ ethyl acetate and $7 \mathrm{~g}$ n-butanol extract. The $\mathrm{n}$-hexane extract $(28 \mathrm{gr})$ was further fractionated by the gravitation of column chromatography and produced 10 fractions (1-10). White needles was found in the fourth fraction, then dissolved with methanol to solve impurities, pure crystal $(485 \mathrm{mg})$ get from recrystallized used hexane and chloroform. Purity test was performed by TLC using hexane and ethyl acetate solvent (4:1) and compared with standard stigmasterol, further determined its melting point, FT-IR and ${ }^{1} \mathrm{H},{ }^{13} \mathrm{C}$ NMR its marked as compound 1 . The melting point was determined using a micro melting point measurement (Fisher Scientific (Fisher Scientific). The hexane fraction (8 to 10) were mixed $(4 \mathrm{~g})$ further fractionated by gravitation column chromatography to get 15 fractions (1-15). The sixth fraction $(18 \mathrm{mg})$ contain a major spot with purple colour. It continued for purification with Sephadex LH-20 column chromatagraphy (Amersham) with

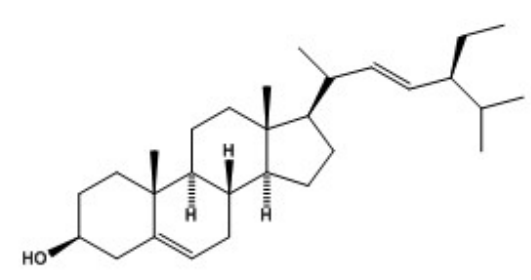

Figure 1. Stigmasterol
Table 1. Primary characteristics of group samples of steroid-sensitive and steroid-resistant nephrotic syndrome [18].

\begin{tabular}{|c|c|c|}
\hline $\begin{array}{c}\mathrm{C} \\
\text { number }\end{array}$ & $\begin{array}{l}\delta{ }^{13} \mathrm{C}-\mathrm{NMR}\left(\mathrm{CDCl}_{3}\right) \\
\text { stigmasterol }(\mathrm{pPm})\end{array}$ & $\begin{array}{c}\delta{ }^{13} \mathrm{C}-\mathrm{NMR} \text { of }\left(\mathrm{CDCl}_{3}\right) \\
\text { compound } 1(\mathrm{ppm})\end{array}$ \\
\hline 1 & 37.2 & 37.43 \\
\hline 2 & 31.6 & 31.84 \\
\hline 3 & 71.8 & 72.01 \\
\hline 4 & 42.5 & 42.47 \\
\hline 5 & 140.9 & $\underline{140.92}$ \\
\hline 6 & 121.9 & $\underline{121.92}$ \\
\hline 7 & 32.8 & 32.08 \\
\hline 8 & 31.9 & 31.84 \\
\hline 9 & 50.2 & 50.32 \\
\hline 10 & 36.6 & 36.70 \\
\hline 11 & 22.7 & 21.40 \\
\hline 12 & 39.7 & 39.85 \\
\hline 13 & 42.3 & 42.45 \\
\hline 14 & 56.9 & 57.04 \\
\hline 15 & 24.3 & 24.55 \\
\hline 16 & 28.9 & 29.13 \\
\hline 17 & 56.0 & 56.10 \\
\hline 18 & 12.0 & 12.24 \\
\hline 19 & 19.3 & 19.59 \\
\hline 20 & 40.5 & 40.72 \\
\hline 21 & 21.3 & 21.30 \\
\hline 22 & $\underline{138.3}$ & $\underline{138.53}$ \\
\hline 23 & 129.3 & 129.43 \\
\hline 24 & 51.2 & 51.42 \\
\hline 25 & 31.8 & 31.84 \\
\hline 26 & 18.9 & 19.16 \\
\hline 27 & 21.1 & 21.32 \\
\hline 28 & 25.4 & 24.55 \\
\hline 29 & 12.2 & 12.24 \\
\hline
\end{tabular}

dichloromethane: methanol (1:1) as a mobil phase. Also get the yield $16 \mathrm{mg}$ pure compound 2. Further determined its melting point, FT- IR and ${ }^{1} \mathrm{H},{ }^{13} \mathrm{C}$ NMR one and two dimension. Ethyl acetate fraction $(2.8 \mathrm{~g})$ were subjected to silica gel G60 column chromatography using mobile phase n-hexane-ethyl acetate and methanol by gradient afforded 13 fractions. The ninth fraction $(53 \mathrm{mg})$ was further purification with Sephadex LH-20 column chromatography with dichloromethane: methanol (1:1) as a mobil phase. Also get the yield $41 \mathrm{mg}$ pure compound 3. Compound 3 deducted by spectroscopic data FT-IR, LC-MS, ${ }^{1} \mathrm{H}$ and ${ }^{1} \mathrm{NMR}$ 1D and 2D. IR spectrum were taken FT-IR Prestige-21, Shimadzu, NMR spectra of ${ }^{1} \mathrm{H},{ }^{13} \mathrm{C}$, $\mathrm{HMQC}$ and $\mathrm{HMBC}$ were measured using an Inova Plus, Unity NMR 500 at $500 \mathrm{MHz}\left({ }^{1} \mathrm{H}\right)$ and $125 \mathrm{MHz}$ $\left({ }^{13} \mathrm{C}\right)$ with TMS as an internal standard in measurement NMR spectrometer. LC-MS analysis was performed using a Mariner spectrometry equipped with a binaru pump. The HPLC was interfaced with a Q-tof 
Table 2. ${ }^{\mathrm{I}} \mathrm{H},{ }^{13} \mathrm{C} \delta$ NMR data, HMQC and HMBC of Compound 2

\begin{tabular}{|c|c|c|c|}
\hline No & $\boldsymbol{\delta}^{13} \mathrm{C}_{\mathrm{PPm}}(\mathrm{DEPT})$ & $\mathrm{HMQC} \boldsymbol{\delta}^{1} \mathrm{H}(\mathrm{pPm})$ & HMBC \\
\hline 1 & $31.40(\mathrm{CH} 2)$ & $2.45(2 \mathrm{H}, \mathrm{m})$ & $\mathrm{C}-31$ \\
\hline 2 & $24.02(\mathrm{CH} 2)$ & $1.72(1 \mathrm{H}, \mathrm{m}) ; 1.92(1 \mathrm{H}, \mathrm{m})$ & $\mathrm{C}-3, \mathrm{C}-4, \mathrm{C}-22$ \\
\hline 3 & $49.41(\mathrm{CH})$ & $4.39(1 \mathrm{H}, \mathrm{q})$ & $\mathrm{C}-1, \mathrm{C} 2, \mathrm{C} 22$ \\
\hline 4 & $55.21(\mathrm{CH})$ & $5.18(1 \mathrm{H}, \mathrm{q})$ & $\mathrm{C}-23$ \\
\hline 5 & 176.77 & & \\
\hline 6 & 95.14 & $8.55(1 \mathrm{H}, \mathrm{s})$ & C-4, C-7, C-8 \\
\hline 7 & 144.25 (C) & & \\
\hline 8 & $131.93(\mathrm{C})$ & & \\
\hline 9 & $137.90(\mathrm{C})$ & & \\
\hline 10 & $142.90(\mathrm{C})$ & & \\
\hline 11 & $103.21(\mathrm{CH})$ & $9.25(1 \mathrm{H}, \mathrm{s})$ & C-9 \\
\hline 12 & $156.39(\mathrm{C})$ & & \\
\hline 13 & $136,73(\mathrm{C})$ & & \\
\hline 14 & $146.07(\mathrm{C})$ & & \\
\hline 15 & 150.19 (C) & & \\
\hline 16 & $107.69(\mathrm{CH})$ & $9.37(1 \mathrm{H}, \mathrm{s})$ & $\mathrm{C}-14, \mathrm{C}-17, \mathrm{C}-18$ \\
\hline 17 & $140.04(\mathrm{C})$ & & \\
\hline 18 & $131.64(\mathrm{C})$ & & \\
\hline 19 & $164.74(\mathrm{C})$ & & \\
\hline 20 & 93.06 (C) & & \\
\hline 21 & $111.63(\mathrm{C})$ & & \\
\hline 22 & $177.74(\mathrm{C})$ & & \\
\hline 23 & $24.02\left(\mathrm{CH}_{3}\right)$ & $1.74(3 \mathrm{H}, \mathrm{d}, \mathrm{J}=7.8 \mathrm{~Hz})$ & $\mathrm{C}-3, \mathrm{C}-4, \mathrm{C}-5$ \\
\hline 24 & $12.11\left(\mathrm{CH}_{3}\right)$ & 3. $33(3 \mathrm{H}, \mathrm{s})$ & C-7, C-8, C-9 \\
\hline 25 & $128.55(\mathrm{CH})$ & $7.83(\mathrm{dd}, \mathrm{J}=10 ; 15 \mathrm{~Hz})$ & $\mathrm{C}-8, \mathrm{C}-13, \mathrm{C}-26$ \\
\hline 26 & $123.87 \mathrm{CH} 2)$ & $6.17(\mathrm{dd}, \mathrm{J}=15 ; 1.5 \mathrm{~Hz}) ; 6.27(\mathrm{dd}, \mathrm{J}=10 ; 1.5 \mathrm{~Hz})$ & $C-9, C-25$ \\
\hline 27 & $11.17\left(\mathrm{CH}_{3}\right)$ & $3.08(3 \mathrm{H}, \mathrm{s})$ & $\mathrm{C}-12, \mathrm{C}-13, \mathrm{C}-14$ \\
\hline 28 & $19.46(\mathrm{CH} 2)$ & $3.51(2 \mathrm{H}, \mathrm{q}, \mathrm{J}=10 ; 5 \mathrm{~Hz})$ & C-13, C-14,C-15, C-29) \\
\hline 29 & $17.54(\mathrm{CH} 3)$ & $1.59(3 \mathrm{H}, \mathrm{t})$ & 146.07, 19.46 (C-14, C28) \\
\hline 30 & $12.45\left(\mathrm{CH}_{3}\right)$ & $3.64(3 \mathrm{H}, \mathrm{s})$ & $140.04,131.64(\mathrm{C}-17, \mathrm{C}-18)$ \\
\hline 31 & $173.43(\mathrm{C}=\mathrm{O})$ & & \\
\hline $1^{\prime}$ & $61.64(\mathrm{CH} 2-\mathrm{O})$ & $4.52(1 \mathrm{H}, \mathrm{q})$ & \\
\hline $2^{\prime}$ & $118.07(\mathrm{CH} 2)$ & $5.23(1 \mathrm{H}, \mathrm{q})$ & $16.51,40.02\left(\mathrm{C}^{4}, \mathrm{C}-17^{\prime}\right)$ \\
\hline $3^{\prime}$ & 142.90 (C) & & \\
\hline $4^{\prime}$ & $40.02\left(\mathrm{CH}_{2}\right)$ & $1.92(2 \mathrm{H}, \mathrm{m})$ & \\
\hline $5^{\prime}$ & $39.53(\mathrm{CH} 2)$ & $1,12(1 \mathrm{H}, \mathrm{m}) ; 1,19(1 \mathrm{H}, \mathrm{m})$ & \\
\hline 6 & $24.59(\mathrm{CH} 2)$ & $1.26(2 \mathrm{H}, \mathrm{m})$ & \\
\hline $7^{\prime}$ & $28.14(\mathrm{CH})$ & $1.50(1 \mathrm{H}, \mathrm{m})$ & \\
\hline $8^{\prime}$ & $24.95(\mathrm{CH} 2)$ & $1.30(2 \mathrm{H}, \mathrm{m})$ & \\
\hline$g^{\prime}$ & $36.83(\mathrm{CH} 2)$ & $1.23(2 \mathrm{H}, \mathrm{m})$ & \\
\hline $10^{\prime}$ & $37.44(\mathrm{CH} 2)$ & $1.11(1 \mathrm{H}, \mathrm{m}) 1.21(1 \mathrm{H}, \mathrm{m})$ & $\mathrm{C}-8^{\prime}, \mathrm{C}-12^{\prime}, \mathrm{C}-19^{\prime}$ \\
\hline $11^{\prime}$ & $32.80(\mathrm{CH})$ & $2.74(1 \mathrm{H}, \mathrm{m})$ & \\
\hline $12^{\prime}$ & 37.49 (CH2) & $1.22(2 \mathrm{H}, \mathrm{m})$ & \\
\hline $13^{\prime}$ & $24.19(\mathrm{CH} 2)$ & $1.26(2 \mathrm{H}, \mathrm{m})$ & \\
\hline $14^{\prime}$ & $37.56 \mathrm{CH} 2)$ & $1.24(2 \mathrm{H}, \mathrm{m})$ & \\
\hline $15^{\prime}$ & $32.96(\mathrm{CH})$ & $2.74(1 \mathrm{H}, \mathrm{m})$ & \\
\hline 16 & $19.90(\mathrm{CH} 3)$ & $0.78(3 \mathrm{H}, \mathrm{d}, \mathrm{J}=7.0 \mathrm{~Hz})$ & \\
\hline $17^{\prime}$ & $16.51(\mathrm{CH} 3)$ & $1.62(3 \mathrm{H}, \mathrm{s})$ & $\mathrm{C}-2^{\prime}, \mathrm{C}-3^{\prime}, \mathrm{C}-4^{\prime}$ \\
\hline 18 & $22.80(\mathrm{CH} 3)$ & $0.84(3 \mathrm{H}, \mathrm{s})$ & $\mathrm{C}-8^{\prime}$ \\
\hline 19 & $22.89(\mathrm{CH} 3)$ & $0.87(3 \mathrm{H}, \mathrm{s})$ & $\mathrm{C}-9^{\prime}, \mathrm{C}-12^{\prime}$ \\
\hline $20^{\prime}$ & $19.84(\mathrm{CH} 3)$ & $0.81(3 \mathrm{H}, \mathrm{d}, \mathrm{J}=6.5 \mathrm{~Hz})$ & $\mathrm{C}-13^{\prime}, \mathrm{C}-14^{\prime}, \mathrm{C}-15^{\prime}$ \\
\hline
\end{tabular}

mass spectrometer fitted with an ESI source. Full-scan mode from m/z 100 to 1200 was performed with a source temperature of $140^{\circ} \mathrm{C}$. HPLC column (Phenomenex $5 \mu$.C18, $150 \times 1 \mathrm{~mm}$ i.d) was used for the analysis. Solvent was methanol with 0,3 acetic acids. Solvent delivered at a total flow rate of 0.05 $\mathrm{mL} / \mathrm{min}$. The solvent is running by isocratic elution.

\section{RESULTS AND DISCUSSION \\ Compound 1}

Compound 1 resulted from hexane fraction white needles $485 \mathrm{mg}$, the melting point is $160-162^{\circ} \mathrm{C}$. The IR $(\mathrm{KBr})$ spectrum data indicate the presence a hydroxyl group (-OH) showed an absorption peak in the region. 3419.8 - $3294.4 \mathrm{~cm}^{-1}$ (broad) and the absorption band at $2937.6-2864.3 \mathrm{~cm}^{-1}$ indicated the presence of $-\mathrm{CH}$ aliphatic asymmetric stretching of $-\mathrm{CH}_{3}$,$\mathrm{CH}_{2}-$ and $>\mathrm{CH}_{2}$ groups. The absorption band at $1643.4 \mathrm{~cm}^{-1}$ indication presence of C-O- stretching. The ${ }^{1} \mathrm{H}-\mathrm{NMR}$ spectroscopy data $\left(\mathrm{CDCl}_{3}, \quad 500 \mathrm{MHz}\right)$ showed chemical shift $(\delta)$ of methyl singlet at 1,01 $\operatorname{ppm}(3 \mathrm{H}, s)$ and $1,03 \mathrm{ppm}(3 \mathrm{H}, s)$, it is also contained three methyl doublet at $0,84 \mathrm{ppm},(3 \mathrm{H} d, j=3,15 \mathrm{~Hz})$; $0,79(3 \mathrm{H}, d, j=5,9 \mathrm{~Hz}) ; 0,83(3 \mathrm{H}, d, j=5,6 \mathrm{~Hz})$, one methyl doublet-doublet at $\delta 0,91 \mathrm{ppm}(3 \mathrm{H}, d d)$.

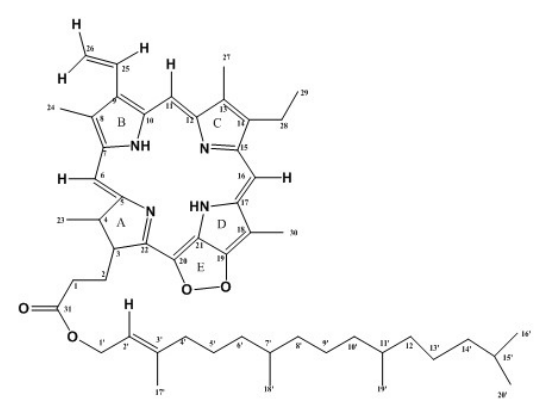

A

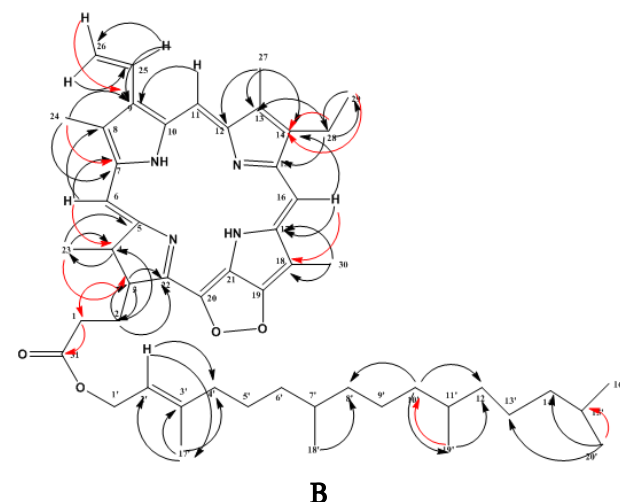

Figure 2. Analoque of Pheophytin (A); Correlation of Analoque pheophytin (B) 
Table 3. Chemical shift of Proton and Carbon NMR and HMBC correlation of Compound 3 ( $\beta$-sitosterol- $D$ glycoside)

\begin{tabular}{|c|c|c|c|}
\hline No & $\delta^{2} \mathbf{H}(\mathrm{pp}=)$ & $\delta^{13} \mathrm{C}(\mathrm{pp}=)$ & HXBC \\
\hline 1 & $1,78(\mathrm{~m}) ; 1,80(\mathrm{~m})$ & 36,86 & \\
\hline 2 & $1.51(\mathrm{~m})$ & 31,41 & \\
\hline 3 & 2,89 (seitet) & 75,49 & \\
\hline 4 & $2.36(d d ; J 2.35 ; 2.35 \mathrm{~Hz})$ & 38.31 & \\
\hline 5 & - & 140,6 & \\
\hline 6 & $5.33(\mathrm{~d}, \mathrm{~J},=5.15 \mathrm{~Hz})$ & 121,27 & $C-1, C-4, C-7, C-8 C-10$ \\
\hline 7 & $1,38(\mathrm{~m}) ; 1,40(\mathrm{~m})$ & 31,44 & \\
\hline 8 & $1,47(b)$ & 31,41 & \\
\hline 9 & $0,99(b)$ & 49,62 & \\
\hline 10 & - & 36,23 & \\
\hline 11 & $1.36(\mathrm{~m}) ; 1.51(\mathrm{~m})$ & 20,62 & \\
\hline 12 & $1,16(m, b)$ & 39.25 & \\
\hline 13 & - & 41,88 & \\
\hline 14 & $1,09(\mathrm{~m})$ & 56,20 & \\
\hline 15 & $1.53(\mathrm{~m})$ & 23.91 & \\
\hline 16 & $1,78(\mathrm{~m}) ; 1,63(\mathrm{~m})$ & 29,29 & \\
\hline 17 & $1,03(\mathrm{~m})$ & 55,43 & \\
\hline 18 & $0,65(s)$ & 11,72 & $C_{-13}, C_{-14}, C_{-17}$ \\
\hline 19 & $0,95(s)$ & 18,95 & $\mathrm{C}-5, \mathrm{C}-7, \mathrm{C}-8, \mathrm{C}-9$ \\
\hline 20 & $1,80(\mathrm{~m})$ & 36,86 & \\
\hline 21 & $0,90(s)$ & 18,65 & $C-15, C-17, C-21$ \\
\hline 22 & $1,02(\mathrm{~m})$ & 33.36 & \\
\hline 23 & $1,15(\mathrm{~m})$ & 25,41 & \\
\hline 24 & $0.91(s)$ & 45,15 & \\
\hline 25 & $1,63(\mathrm{~m})$ & 28,70 & \\
\hline 26 & $0,80(d, J=3,2 \mathrm{~Hz})$ & 19,14 & $C-24, C-25, C-27$ \\
\hline 27 & $0,83(d, J=3,2 \mathrm{~Hz}\rangle$ & 19,76 & $\mathrm{C}_{-26}, \mathrm{C}_{29}$ \\
\hline 28 & $1.25(\mathrm{~m})$ & 22,63 & \\
\hline 29 & $0,82(t)$ & 1182 & $\mathrm{C}-26, \mathrm{C}-28$ \\
\hline $1^{\prime \prime}$ & $4,22(1 \mathrm{H}, d, J=7,8 \mathrm{~Hz})$ & 100,77 & $\mathrm{C}-2^{\prime}, \mathrm{C}-3^{\prime \prime}$ \\
\hline$z^{\prime}$ & $3,11(1 \mathrm{H}, \mathrm{m})$ & 76,79 & $\mathrm{C}^{*}$ \\
\hline $3^{\prime}$ & $3,47(1 \mathrm{H}, \mathrm{m})$ & 76.91 & $C-1^{\prime}, C-4^{\prime \prime}$ \\
\hline $4^{4}$ & $3,06(1 \mathrm{H}, \mathrm{m})$ & 70,11 & \\
\hline $5^{\prime}$ & $3,47(1 \mathrm{H}, \mathrm{m})$ & 76.91 & \\
\hline $6^{\prime \prime}$ & $3,64(1 \mathrm{H}, \mathrm{m}) ; 3.39(1 \mathrm{H}, \mathrm{m})$ & 61,10 & $C-4^{*}$ \\
\hline
\end{tabular}

showed one olefinic proton substitution at $\delta 5,35 \mathrm{ppm}$ $(1 \mathrm{H}, d . J=5,0 \mathrm{~Hz}, \mathrm{H}-6)$ and two protons with substituted olefinic at $\delta 5,16(1 \mathrm{H}, t, J=8,4 \mathrm{~Hz}, \mathrm{H}-22)$ and $5,01(1 \mathrm{H}, t, J=8,4 \mathrm{~Hz}, \mathrm{H}-23)$. Chemical shift at $\delta 3,52$ ppm $(1 \mathrm{H}, m)$ showed an axial oxime thine forward oriented ( $\beta$ ) equatorial of hydroxy group at $\mathrm{C}-3$. The presence of abundants spectra at $\delta 1,11-2,3 \mathrm{ppm}$ showed the presence of $\mathrm{sp}^{3}$ bonds from methylene and methine groups. The ${ }^{13} \mathrm{C}$ NMR of compound one ( $\mathrm{Ta}-$ ble 1) showed there are 29 carbons in the molecule. There is presence three olefinic resonance at $\delta 121.92$ ppm, $138.53 \mathrm{ppm}$ and $129.43 \mathrm{ppm}$ correspondent to C6, C-22 and C-23 and a signal at $\delta 140.92 \mathrm{ppm}$ correspondent of carbon quarter of $\mathrm{C}-5$. On the basis of ${ }^{1} \mathrm{H}$ and ${ }^{13} \mathrm{C}$ NMR spectral data and compared with an authenthic compound that compound 1 were identified and established stigmasterol (Figure 1).

\section{Compound 2}

Compound 2 resulted from $n$-hexane fraction about $16 \mathrm{mg}$, dark violet amorf powder. The IR spectrum of compound 2 showed the presence of an -OH group $\left(3,342.64 \mathrm{~cm}^{-1}\right)$ and carbonyls group $\left(1,743.65 \mathrm{~cm}^{-1}\right.$ and $\left.1602.85 \mathrm{~cm}^{-1}\right)$. Its molecular formula is $\mathrm{C}_{51} \mathrm{H}_{70} \mathrm{~N}_{4} \mathrm{O}_{4}$, $(\mathrm{m} / \mathrm{z}=803.06)$ FAB-MS. Result from ${ }^{1} \mathrm{H}$ NMR, there were seven methyls at $\delta 3.63,3.33,3.08 \mathrm{ppm}$ (each $s$ ), $1.74(\mathrm{~d}, \mathrm{~J}=5.0 \mathrm{~Hz}), 1.59(\mathrm{t}, \mathrm{J}=8.3 \mathrm{~Hz}), 0.81(\mathrm{~d}, \mathrm{~J}=6.5$ $\mathrm{HZ}), 0.78(d, J=6.5 \mathrm{~Hz})$; three olefinic singlets at $\delta$ 9.37, 9.25, $8.55 \mathrm{ppm}$; one vinyl group or exo methylene at $\delta 7.83(d d, J=15$ and $10 \mathrm{~Hz}), 6.27(d d, J=10 ; 1.3$ $\mathrm{Hz})$ with methyl couple at $6.17(d d, J=15 ; 1.5 \mathrm{~Hz})$ indicate its trans oriented. Compound 2 differed from a known compound pheophytin 1 [14]. In the position of C-31, where the ethyl esters group in the pheophytin 1 was replaced by an phytyl ester in compound 2 which shown in the spectra $\left(\delta{ }^{1} \mathrm{H}\right.$ and $\delta$ $\left.{ }^{13} \mathrm{C}\right)$ NMR C1'-20' at Table 2. ${ }^{13} \mathrm{C}$ NMR spectra compound 2 is similar with pheophytin, [15], except on ring E peroxide functionalities moiety on C19-21 of ring D. Along with the corresponding NMR spectra (Table 2) from HMQC and HMBC indicate that compound 2 was analogue with pheophytin (Figure $2 \mathrm{~A}$ and 2B).

\section{Compound 3}

Compound 3 was isolated as a white powder from hexane fraction, melting point $140-141^{\circ} \mathrm{C}$. The IR

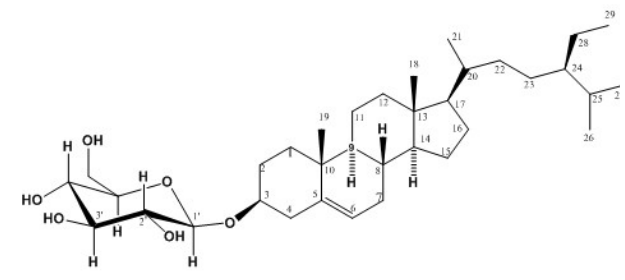

A

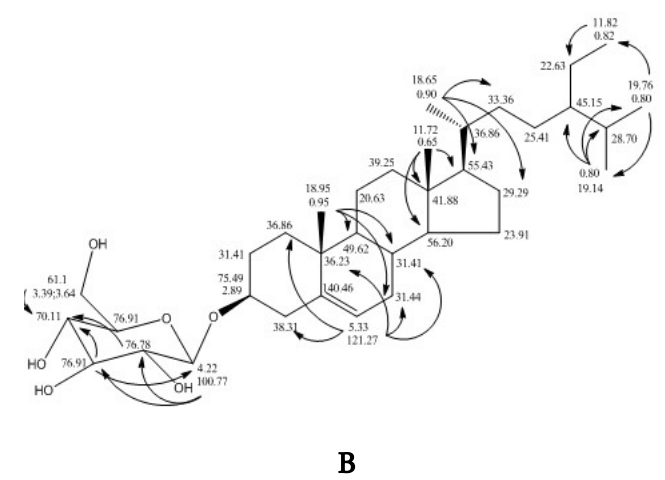

Figure 3. $\beta$-sitostrol-D-glicopyranoside (A); HMBC correlation of $\beta$-sitostrol-D-glicopyran (B) 
spectrum showed an absorption peak in the region (3194- 3358) $\mathrm{cm}^{-1}$. It is indicating the present of a hydroxyl group and the absorption band at (2872-2933) $\mathrm{cm}^{-1}$. It indicated the presence of $-\mathrm{CH}$ aliphatic asymmetric stretching of $-\mathrm{CH}_{3},-\mathrm{CH}_{2}-$ and $>\mathrm{CH}_{2}$ groups. Its molecular formula is $\mathrm{C}_{35} \mathrm{H}_{60} \mathrm{O}_{6} 577\left[\mathrm{M}+\mathrm{H}^{+}\right]$ (LC-MS/ $\mathrm{m} / \mathrm{z}$ ). From the ${ }^{1} \mathrm{H}$ NMR (in DMSO D6) spectrum showed the tertiary methyl $(\delta)$ at $0.65 \mathrm{ppm}$ (Me-18) and 0.95 ppm (Me-19). Three secondary methyls at $0.90 \mathrm{ppm}(\mathrm{Me}-21), 0.80 \mathrm{ppm}(d, J=3.2 \mathrm{~Hz}$, Me-26), $0.83 \mathrm{ppm}(d, J=3.2 \mathrm{~Hz}, \mathrm{M})$. Its presence of anomeric proton at $4.22 \mathrm{ppm}\left(1 \mathrm{H}, d, J 7.8 \mathrm{~Hz}, \mathrm{H}-1^{\prime}\right)$ reflected that the proton is axial-axial to $\mathrm{H}-2$ ' with means glucopyranoside moiety binds to sterol moiety is $\beta$ position $[16,17]$ and one proton olefinic substitution at $\delta 5.33 \mathrm{ppm}(1 \mathrm{H}, d J=5.15 \mathrm{~Hz}, \mathrm{H}-6)$. The ${ }^{13} \mathrm{C} \mathrm{NMR}$ spectra compound 3 revealed presence of 35 carbon atoms in the molecules. The anomeric carbon signal at $\delta 100,77 \mathrm{ppm}$ (C-1') indicated the presence of a single monosacharide moiety. The four methine resonances at $\delta 76.79,76.91,70.11$ and $76.91 \mathrm{ppm}$ as well as methylene resonance at $\delta 61.10 \mathrm{ppm}$ were done C-2', C-3', C4', C5' and C-6', respectively of the $\beta$ - $D$-glucopyranoside. The olefinic resonance at $121.27 \mathrm{ppm}$ corresponded to C-6. The relationships in the bonding structure were proven through long-range correlation of ${ }^{1} \mathrm{H}-\longrightarrow{ }^{13} \mathrm{C}$ of $\mathrm{HMBC}$ spectrum were showed (Table 3). On the basis of IR, ${ }^{1} \mathrm{H}-\mathrm{NMR}$ and ${ }^{13} \mathrm{C}$ - NMR spectra data and the other physical properties the isolate pure compound 3 were identified and established as $\beta$-sitosterol- $D$-glucopyranoside as shown in Figure 3.

\section{CONCLUSIONS}

From the isolation and characterization of the compounds from hexane and ethyl acetate fraction of ethanol extract Peperomia pellucida L. were resulted three compounds are stigmasterol (1), analogue pheophytin (2) and $\beta$-sitosterol- $D$-glucopyranoside (3)

\section{ACKNOWLEDGMENT}

This research was supported by Competitive "Drug and Molecular Farming" Project funded by Indonesia Institute of Sciences

\section{REFERENCES}

1. Bojo AC, Albana-Gracia E, Poesidio GN (1994) The antibacterial activity of Peperomia pellucida (L) HBK (Piperaceae). Asia life Sciences. 3: 35-44.

2. Joaquim de CB, Mara Silvia PA, Adolfo HMl, Alberto CA, Williams CC (2000) A dimeric ArC2 compound from Peperomia pellucida. Phytochemistry. 55: 779 -782.
3. Khan MR, AD Omoloso (2002) Antibacterial activity of Hygrophila stricta and Peperomia pellucida. Fitoterapia. 73: 251-254.

4. Arrigoni-Blank MdF, Oliveira RLB, Mendes SS, Silva PdA, Antoniolli ÂR, Vilar JC, Cavalcanti SCdH and Blank AF (2002) Seed germination, phenology, and antiedematogenic activity of Peperomia pellucida (L.) H. B. K. BMC Pharmacology. 2: 1-8.

5. Azyba PI, A Adedeji, M Ekor, O Adeyemi (2001) Analgesic activity of Peperomia pellucida aerial parts in mice. Fitoterapia. 72: 57-58.

6. Arrigoni-Blank MdeF, Dmitrieva EG, Franzotti EM, Antoniolli AR, Andrade MR, Marchioro M (2004) Anti-inflammatory and analgesic activity of Peperomia pellucida (L.) HBK (Piperaceae). Journal of Ethnopharmacology. 91: 215-218.

7. Der-Jiun O, Shahid I and Maznah I (2012) Proximate Composition, Nutritional Attributes and Mineral Composition of Peperomia pellucida L. (Ketumpangan Air), Grown in Malaysia. Molecules. 17: 11139-11145.

8. Wei LS, Wee W, Siong JYF, and Syamsumir DF (2011) Characterization of Anticancer, Antimicrobial, Antioxidant Properties and Chemical Compositions of Peperomia pellucida Leaf Extract. Acta Medica Iranica. 49(10): 670674.

9. Xu S, Na L, Meng-Meng N, Cai-Hong Z, Qiao-Rong Y, and Ming-Wei W (2006) Bioactive Compounds from Peperomia pellucida. Journal of Natural Products. 69(2): 247-50.

10. Bayma J de C, Arruda MSP, Müller AH, Arruda AC, Canto WC (2000) A Dimeric ARC2 Compound from Peperomia pellucida. Phytochemistry. 55: 779-782.

11. de Lira PN, da Silva JK, Andrade EH, Sousa PJ, Silva NN, Maia JG (2009) Essential oil composition of three Peperomia species from the Amazon, Brazil. Nat Prod Commun. 4(3): 427-30.

12. Rojas-Martinez R, Arrieta J, Cruz-Antonio, Arrieta-Baez D, Velazquez-Mendes AM and Sanchez-Mendoza ME (2013) Dillapiole, Isolated from Peperomia pellucida, show Gastroprotector Activity againts Ethanol-Induced Gastric Lesion in Wistar Rats. Molecules. 18: 1132711337.

13. Ngueguim fT, Khan MP, Donfack JH, Tewari D, Dimo T, Kamtchouing P, Maurya R, Chamttopadhyay (2013) Ethanol Extract of Peperomia pellucida (Piperaceae) Promote Fracture Healing by an Anabolic Effect on Osteoblasts. Journal of Ethnopharmacology. 148: 62-68.

14. Li H, Lina L, Zheng Q, Kuroda C, and Wang Q (2012) Phaeophytin Analogues from Ligularia knorringiana. Molecules. 17: 5219-5224.

15. Lee TH, Lu CK, Kuo YH, Lo JM, and Lee CK (2008) Un- 
expected Novel Pheophytin Peroxides from the Leaves of Biden pilosa. Helvetica Chimica Acta. 91: 79 - 84.

16. Bai H, Li S, Yin F and Hu L (2005) Isoprenylated naptoquinone dimers firnianones $\mathrm{A}, \mathrm{B}$ and $\mathrm{C}$ from Firniana plantanifolia. J. Nat. Prod. 60: 1159-1163.
17. Silverstein RM, Bassler GC, and Morrill TC (1991) Spectrometric Identification of Organic Compounds. Singapore, 221.

18. Goal LJ and Akihisa T (1997) Analysis of Sterols. London and New York: Blackie Academic \& Professional. 